\title{
On-off transition and ultrafast decay of amino acid luminescence driven by modulation of supramolecular packing
}

\author{
Zohar A. Arnon, Topaz Kreiser, Boris Yakimov, Noam Brown, Ruth Aizen, Shira Shaham-Niv, \\ Pandeeswar Makam, Muhammad Nawaz Qaisrani, Emiliano Poli, Antonella Ruggiero, Inna \\ Slutsky, Ali Hassanali, Evgeny Shirshin, Davide Levy and Ehud Gazit*
}

\author{
Z. A. Arnon, T. Kreiser, N. Brown, R. Aizen, Dr. S. Shaham-Niv, Dr. P. Makam, Prof. E. Gazit \\ Department of Molecular Microbiology and Biotechnology, George S. Wise Faculty of Life Sciences, Tel Aviv \\ University, Tel Aviv 6997801, Israel. \\ E-mail: ehudga@tauex.tau.ac.il
}

Dr. B.Yakimov, Dr. E. Shirshin

Faculty of Physics, M.V. Lomonosov Moscow State University, Moscow, 119991 Russia.

World-Class Research Center "Digital biodesign and personalized healthcare", Sechenov First Moscow State Medical University, 119991, Moscow, Russia.

Dr. M. N. Qaisrani, Dr. E. Poli, Dr. A. Hassanali

International Centre for Theoretical Physics, Strada Costiera, 11, 34151, Trieste, Italy.

Dr. A. Ruggiero, I. Slutsky

Department of Physiology and Pharmacology, Sackler Faculty of Medicine, Tel Aviv University, Tel Aviv University, 6997801, Tel Aviv, Israel.

Dr. D. Levy

X-Ray Diffraction Lab, Wolfson Applied Materials Research Centre, Tel Aviv University, Tel Aviv 6997801, Israel.

Prof. E. Gazit

Department of Materials Science and Engineering Iby and Aladar Fleischman Faculty of Engineering, Tel Aviv University, Tel Aviv 6997801, Israel.

Keywords: amino acids, aggregation induced fluorescence, optical properties, crystal packing, reversible autofluorescence, ultrafast lifetime decay

\section{Abstract}

It has been experimentally observed that various biomolecules exhibit clear luminescence in the visible upon aggregation, contrary their monomeric state. However, the physical basis for this phenomenon is still elusive. Here, we systematically examine all coded amino acids to provide non-biased insights into this phenomenon. Several amino acids, including non-aromatic, show intense visible luminescence. While lysine crystals display the highest signal, the very chemically similar non-coded ornithine does not, implying a role for molecular packing rather than the chemical characteristics of the molecule. Furthermore, cysteine show luminescence that is indeed crystal-packing-dependent as repeated rearrangements between two crystal structures result in a reversible on-off optical transition. In addition, ultrafast lifetime decay is experimentally validated, corroborating a recently raised hypothesis regarding the governing 
role of $n \pi^{*}$ states in the emission formation. Collectively, our study supports the hypothesis that electronic interactions between molecules that are non-fluorescent and non-absorbing at the monomeric state may result in reversible optically-active states by the formation of supramolecular fluorophores.

\section{Introduction}

In nature, proteins and peptides offer a broad range of characteristics and attributes, which provide an extensive variety of mechanical, electrical and optical properties. These are often derived from the physicochemical properties of the amino acid building blocks that constitute the proteinaceous polymer. In addition to the amino acid content, the arrangement of the protein in its environment, i.e., secondary, tertiary and quaternary structure, can dramatically influence the resulting physical properties. This arrangement is affected by factors such as the solvent, temperature, ionic strength, etc. Recently, several studies described the intrinsic luminescence in the visible range of protein assemblies, while the monomeric protein in solution is not luminescent. ${ }^{1,2}$ Consistently, it is now evident that some amyloids, once assembled, exhibit fluorescence that is not demonstrated in the monomeric state in solution. ${ }^{3-6}$

Monomeric amino acids were subjected to extensive research over the years. Recently, a great emphasis was given to the ability of amino acids to serve as building blocks for the formation of various supramolecular assemblies with attractive features. ${ }^{7-9}$ It was shown that single amino acids in their aggregated form can also produce a fluorescent signal, which is not evident in the monomeric state. ${ }^{10-13}$ Numerous observations suggest that the optical phenomenon of aggregation induced intrinsic fluorescence is broader than originally speculated, as it also applies to many other metabolites and nucleic acids. ${ }^{14-19}$ Circumstantial evidence and argumentation suggested that supramolecular packing is important. It was suggested that the hydrogen bonds and, in specific cases, the aromatic stacking, can underlie these optical properties. ${ }^{1,20}$ Yet, other views have suggested impurities and oxidation as alternative mechanisms, ascribing no significant role to the molecular arrangement. ${ }^{21}$ Indeed, 
oxidation of aromatic moieties is known to result in the formation of fluorescent products. However, this cannot readily explain the emergence of intrinsic fluorescence upon aggregation of non-aromatic species. ${ }^{6,19,20}$ Overall, the key evidence to distinguish between these options and elucidate the mechanism of intrinsic fluorescence upon aggregation was so far missing. We believe that the reversible transitions between the luminescent and non-luminescent states upon controllable change of aggregate structure is such an evidence. In this work, we aimed at designing and performing such an experiment.

The phenomenon of aggregation-dependent luminescence of proteins is a rapidly evolving field. ${ }^{1,2,17,22-24,3-5,10-13,16}$ Protein aggregates were shown to absorb light at wavelengths above $300 \mathrm{~nm}$ and to exhibit a structure-specific fluorescence in the visible range, even in the absence of aromatic amino acids. ${ }^{6}$ A plausible explanation for this phenomenon is the formation of structure-specific supramolecular fluorophores that are permissive to proton transfer across hydrogen bonds. ${ }^{1,25,26}$ Other hypotheses postulate that the fluorescence is associated with electron-hole recombination due to charge transfer between charged amino acids ${ }^{5,22,27}$ or that deep-blue autofluorescence stems from carbonyl double bonds of the protein backbone. ${ }^{11}$ Following these studies, we have decided to look into the intrinsic optical properties of single amino acid and metabolite assemblies. Indeed, we have shown that adenine, phenylalanine, tyrosine and tryptophan exhibit autofluorescence in the visible range upon self-assembly. ${ }^{10}$ This allows the detection of metabolite assemblies within living cells, without any use of external dyes that could interfere with the ability to accurately model a given sample. ${ }^{28}$ Other derivatives of tyrosine were also shown to exhibit luminescence upon aggregation. ${ }^{29}$ Yet, the underlying mechanism of the autofluorescence of these assemblies is controversial, as in all cases it can be attributed to the aromatic moiety. In addition, the explanation of impurities as the source of fluorescence is still a plausible option. Here, we have endeavored to study the optical properties of all 20 coded amino acids in a systematic, non-biased approach. Each amino acid was optically characterized in two states: the original powder form, as obtained from the commercial supplier, 
and after being dissolved in heated water and allowed to cool down for recrystallization. Aiming to simplify the process of assembly and in order to eliminate as many factors as possible, we avoided altering solvents, salt concentrations, changing $\mathrm{pH}$, etc. The systematic evaluation of all amino acids, before and after recrystallization, provides mechanistic insights into the broad phenomenon of assembly-dependent intrinsic fluorescence.

\section{Results}

First, we examined the optical properties of the crystalline powders of all 20 coded amino acids in the dried powder form. In addition, each powder was dissolved in water at a high concentration (Table S1). In order to completely dissolve the powders, the aqueous solution was heated to $90{ }^{\circ} \mathrm{C}$ and vortexed until a clear, transparent solution was achieved. The amino acid solutions were then cooled down gradually to room temperature and incubated for several days to allow the formation of assemblies within the solution. The samples were then lyophilized to attain a dried powder of the amino acid assemblies. The optical properties of these powders were examined as well.

The brightfield and confocal fluorescence images obtained under excitation of $405 \mathrm{~nm}$ for all 20 amino acids, as originally received (OS, original Sigma) and after dissolution and reassembly (DR, dissolved and reassembled), as well as quantification of the fluorescence signals, are displayed in Figure 1 (see Figure S1 for spectra). For all samples, similar excitation and detection parameters were used. Based on the current measurements there is no systematic trend between the optical properties and the polarity of the amino acids. However, it does appear as though the charged amino acids tend to be characterized by stronger fluorescence whereas those amino acids with hydrocarbon side chains with methyl groups tend to exhibit very weak optical activity. 

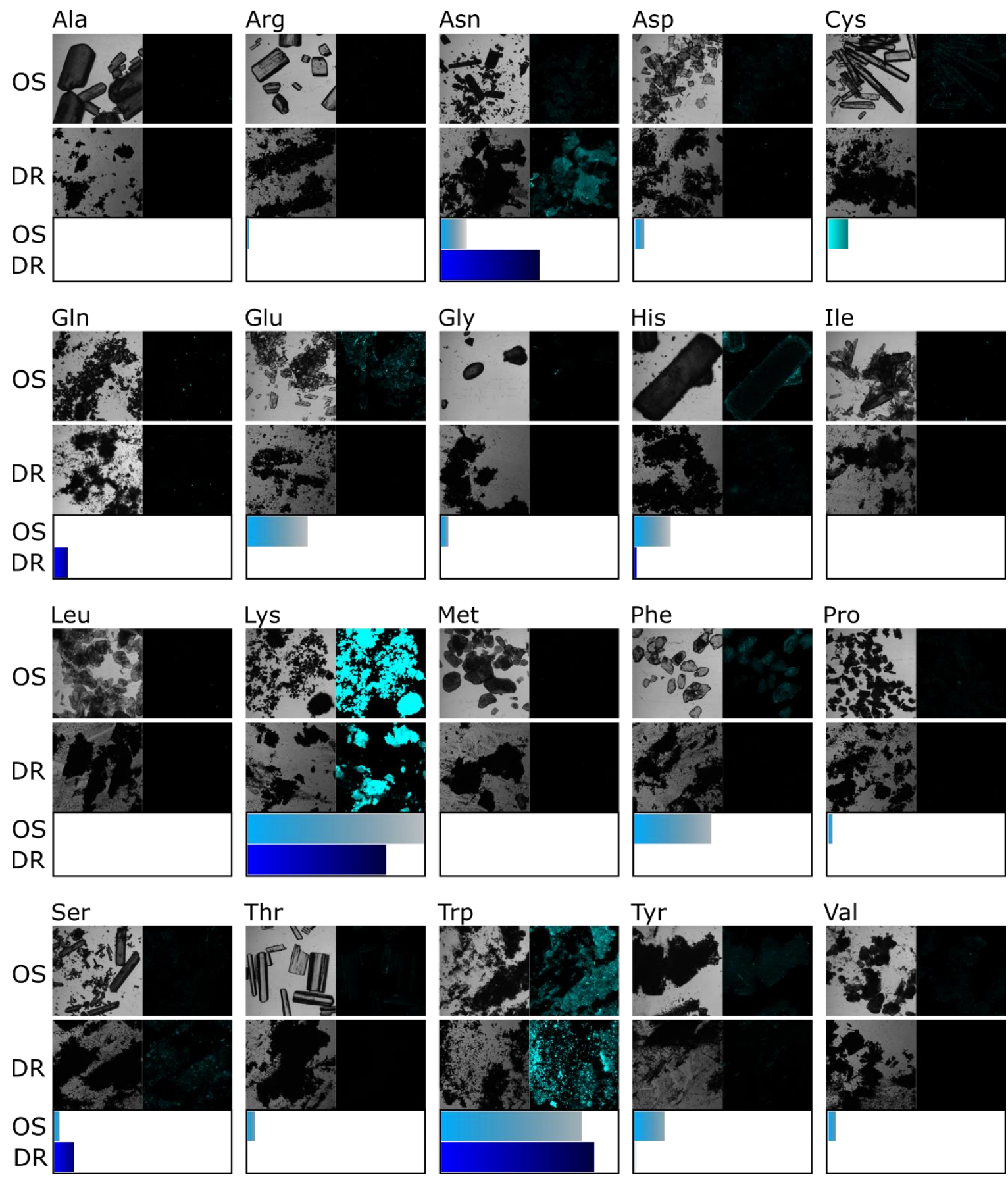

Figure 1. Fluorescence of the 20 coded amino acids. Images of all 20 amino acids, both brightfield (left) and confocal fluorescence at excitation wavelength of $405 \mathrm{~nm}$ (right). Amino acids samples as originally obtained from Sigma-Aldrich are displayed in rows marked with OS (Original Sigma). Amino acids dissolved, reassembled and lyophilized are displayed in rows marked with DR (Dissolved and Reassembled). At the bottom of each amino acid panel are the normalized fluorescence signals of the OS sample (light blue) and the DR sample (dark blue).

We then explored the intense fluorescence of L-lysine, which exhibited the brightest signal of all 20 amino acids. We first inquired whether the length of the amine residue chain of lysine, which has four carbons, plays a role in the optical properties of the crystal. For this purpose, we explored the fluorescence of three additional molecules derived from lysine: L-ornithine, L2,4-diaminobutyric acid and DL-2,3-diaminopropionic acid, which comprise 3, 2 and 1 side 
chain carbons, respectively (Figure 2a). The rationale of this experiment was to alter the crystal packing by shortening the amine residue, thereby modulating the electronic interactions by varying the distance between molecules, and to examine any effect on the resulting optical properties.

All four samples (including lysine) are comprised of small crystalline powders (Figure 2b). The fluorescent signal from each sample was obtained using confocal microscopy, similar to the amino acids shown in Figure 1. Intense fluorescent signals were prominent in the molecules comprising 1-, 2- and 4-carbon chains, while in the 3-carbon chain molecule, ornithine, no visible signal was detected (Figure 2c). The powder X-Ray diffraction (PXRD) patterns of the samples were further examined in order to understand the molecular arrangement of the crystals, which might provide insight into the favorable interactions allowing the crystal fluorescence (Figure 2d). The crystal structures of lysine, ornithine and diaminopropionic acid were previously published. ${ }^{30-32}$ The crystal structure of diaminobutyric acid was determined by the PXRD pattern obtained in this study CCDC Deposition \# 1990651 (see Experimental Section). The results revealed the crystal packing of all four samples, allowing molecular inspection of the optical phenomenon. A possible explanation lies in the type of hydrogen bond network that is formed within the supramolecular structure. The $\mathrm{NH}_{2}$ group of lysine hemihydrate and diaminobutyric acid, and the $\mathrm{NH}_{3}{ }^{+}$of diaminopropionic acid, may interact with a water or chloride ions and donate hydrogen bonds to the molecule complexed in the supramolecular structure. On the other hand, in the case of ornithine, the $\mathrm{NH}_{2}$ groups do not form hydrogen bonds that are donated from the amine group to either a chloride ion or a water molecule in the complex. Thus, the differences in the supramolecular packing involving the lysine side-chains, chloride ions and water molecules can lead to differences in the optical properties. We believe that the packing of the crystal is an important factor in determining its optical behavior, much like the properties of microenvironments such as different solvents, ionic strength, polarity or molecular concentration could be significant for structure-function 
attributes.

Following this line of evidence, we focused on possible interconnections between the optical and structural properties of amino acid powders.

a

\section{L-Lysine}<smiles>NCCCCC(N)C(=O)O</smiles>
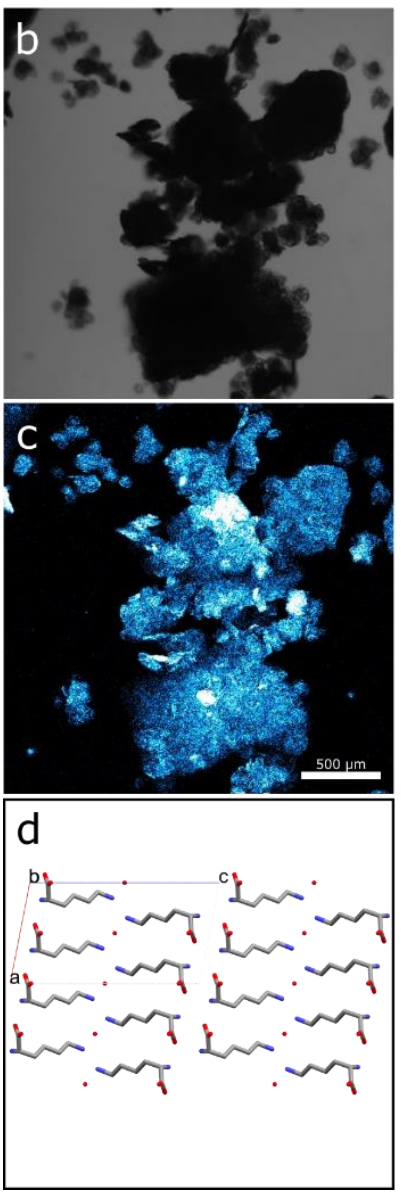

L-Ornithine<smiles>NCCCC(N)C(=O)O</smiles>
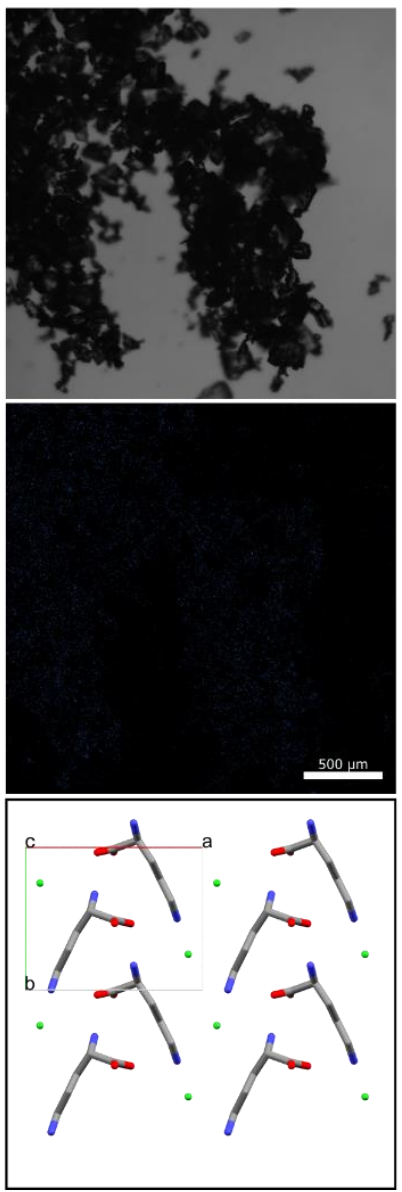

L-2,4-Diaminobutyric acid<smiles>NCCC(N)C(=O)O</smiles>
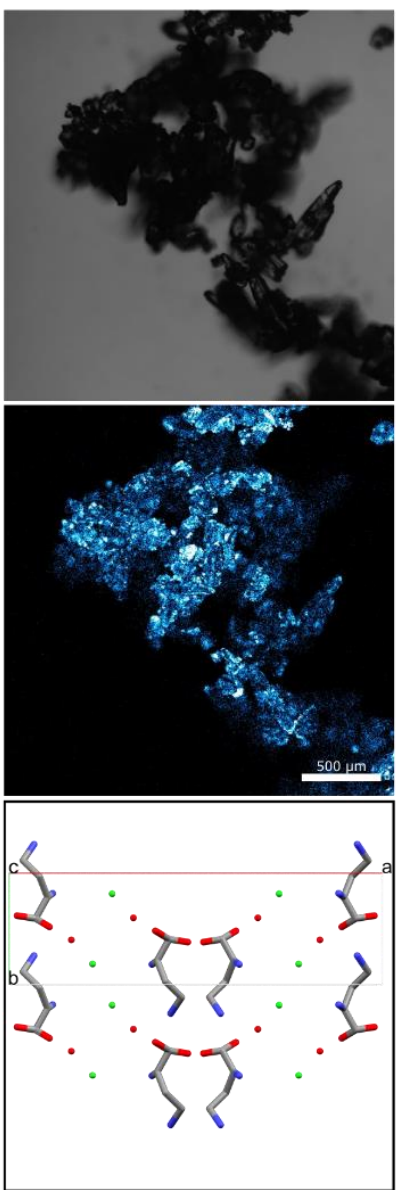

DL-2,3-Diaminopropionic acid<smiles>NCC(N)C(=O)O</smiles>
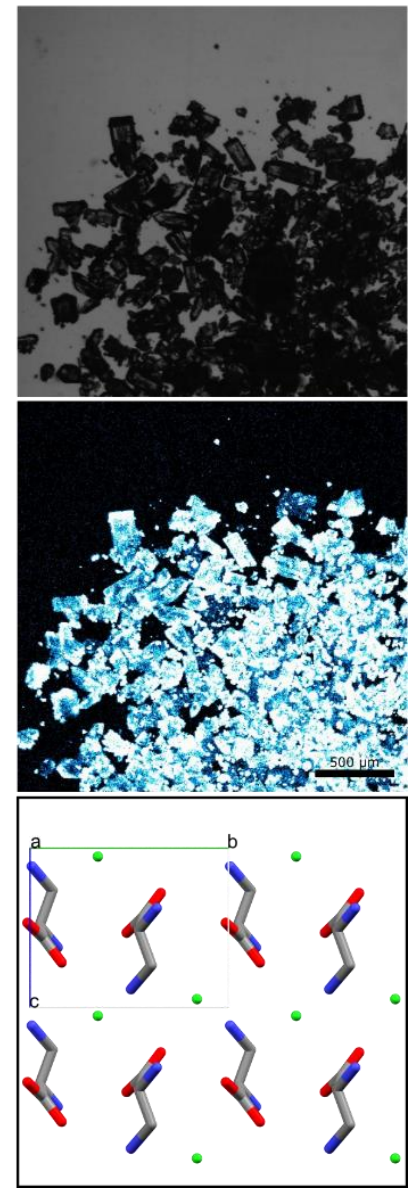

Figure 2. Fluorescence of lysine and its derivatives. a. The chemical scheme of lysine and its derivatives comprising shorter carbon chains. b, c. Microscopy images showing (b) brightfield and (c) fluorescence (excitation at $405 \mathrm{~nm}$ ) analyses of the four powders. Pixel color represents the intensity; black-non, blue-dim, white-strong. d. Crystalline structures of lysine and its derivatives as determined using PXRD.

Next, each of the 20 amino acid samples was examined using PXRD to determine the crystalline structure. The results are summarized in Table S2. Most amino acid crystals were composed of the same crystal structure in both the OS and the DR samples. Out of the 20 amino acids, only cysteine and serine displayed different powder diffraction between their respective OS and DR samples, with a single crystal packing observed in each sample. However, both 
serine samples exhibited some level of fluorescence, while the cysteine DR sample did not show any detectable fluorescence (Figure 1), making the differentiation between the cysteine samples much more straightforward. In addition, the DR serine sample was found to comprise a hemihydrate crystal packing (Table S2), adding another factor of complexity, while both cysteine crystals showed different arrangements of cysteine alone, with four molecules in both unit cells. Hence, we further investigated the OS and DR samples of cysteine (Figure 3a,b), which showed a clear difference in the fluorescent signal (Figure 3c) and exhibited an orthorhombic and monoclinic packing, respectively (Figure 3d,e). ${ }^{33,34}$ Since the process of attaining the OS powder is unknown to us, we have decided to crystallize an orthorhombic cysteine crystal after dissolving the amino acid in order to control the entire procedure. We were able to crystallize both crystal packings, orthorhombic and monoclinic, depending on the crystallization temperature, $4{ }^{\circ} \mathrm{C}$ and $25{ }^{\circ} \mathrm{C}$ respectively, as the only difference in the crystallization conditions. This allowed a reversible crystallization of both crystal packings, exemplifying the significance of the crystal packing to the optical properties of the crystal. Thus, the fluorescent OS powder (Figure 3f) was dissolved and recrystallized at $25^{\circ} \mathrm{C}$ to obtain the non-fluorescent monoclinic DR sample (Figure 3g), and then re-dissolved and recrystallized at $4{ }^{\circ} \mathrm{C}$ to form yet again the fluorescent orthorhombic crystals (Figure $3 \mathrm{~h}$ ). To confirm that the fluorescence does not stem from impurities, the non-fluorescent DR sample was washed several times, to remove any impurities that may reside in the supernatant, before recrystallizing as orthorhombic crystals. Although the fluorescent signal of cysteine is relatively low, a difference in the fluorescent signal between the two crystal packings is clearly evident. A comparison of the two structures shows that there is a subtle difference in the proximity of the $\mathrm{S}-\mathrm{H}$ bonds relative to each other. In the orthorhombic structure, the sulfide groups are packed closer to each other and may facilitate charge transfer, as seen in previous studies. ${ }^{1,6,22}$ It is important to note that the overall macroscopic morphology is dictated by other factors in addition to the packing, as evidenced by different morphologies for an identical packing (compare Figure $3 \mathrm{f}$ 
to $3 \mathrm{~h}$ ), and vice versa (compare Figure $3 \mathrm{~g}$ to $3 \mathrm{~h}$ ). For this reason, the crystal packing was determined using PXRD for each sample and for every iteration. Morphologically, the recrystallized orthorhombic structures (Figure 3h) are similar to the non-fluorescent monoclinic structures (Figure 3g), and are dissimilar to the relatively large OS crystals (Figure 3f); hence, this affirms that the difference in fluorescence is not derived from any morphological discrepancy. As stated above, the crucial experiment to prove the influence of crystal packing on intrinsic fluorescent formation could be an observation of reversible transitions between the luminescent and non-luminescent states upon controllable change of the crystal structure. We believe that the observed on-off switching of cysteine fluorescence upon changing its crystal packing (Figure 3) represents such an experiment.
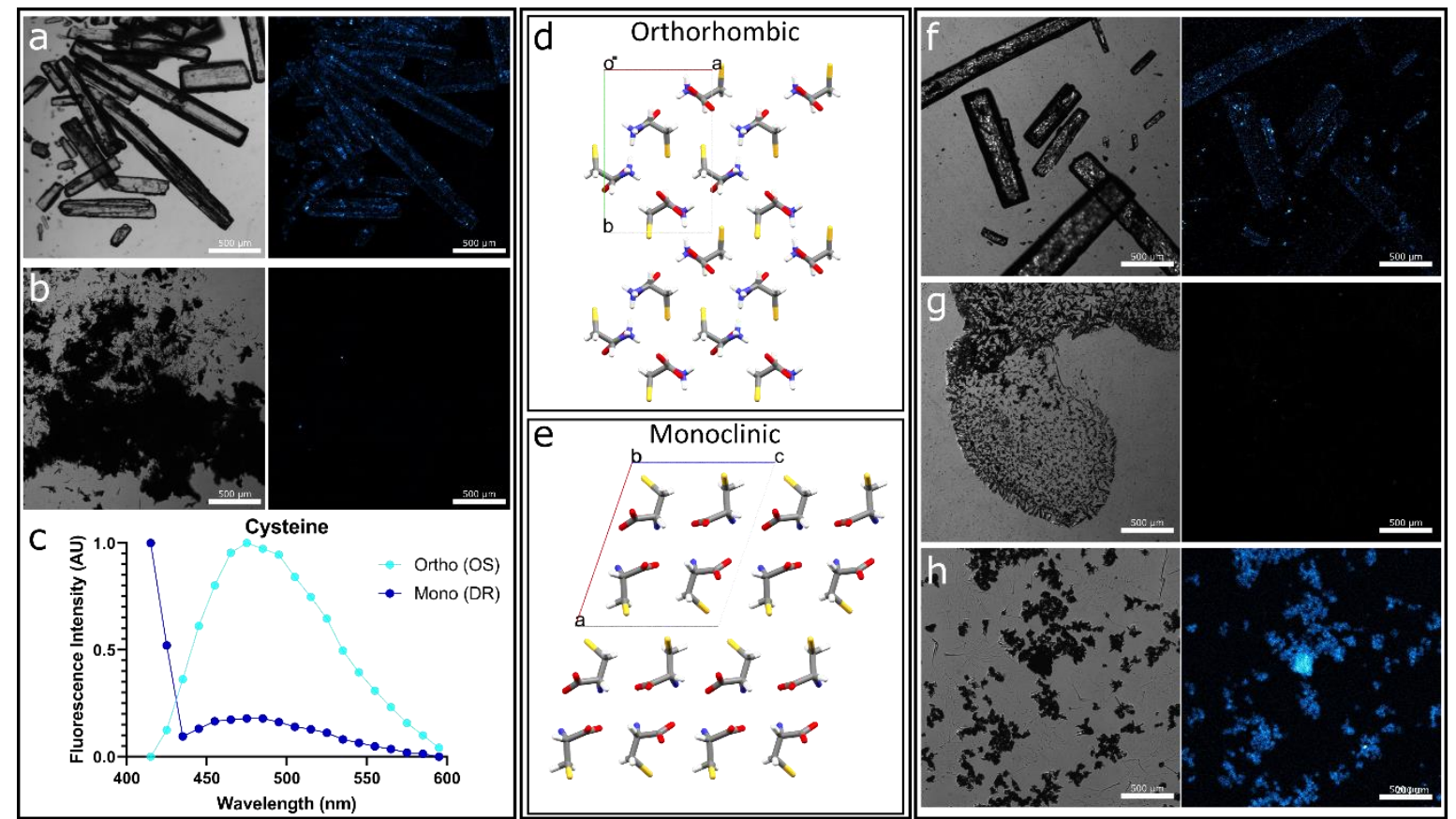

Figure 3. Fluorescence of cysteine. a, b. Confocal microscopy images of the cysteine (a) OS sample and (b) DR sample. c. The normalized fluorescence intensity of cysteine samples as a function of the emission wavelength (excitation at $405 \mathrm{~nm}$ ). d, e. Crystalline structures of cysteine based on PXRD analysis showing (d) the orthorhombic packing of the OS sample and (e) the monoclinic packing of the DR sample. f-h. Confocal imaging of the cysteine (f) OS sample, (g) DR sample and (h) DR sample which was re-dissolved and recrystallized at different conditions to regain an orthorhombic packing. For confocal images, pixel color represents the intensity; black-non, blue-dim, white-strong.

In an attempt to explain the underlying mechanism of the self-assembly induced fluorescence of amino acids, we tried to find a common thread in the aggregation induced fluorescence related literature. First principles quantum chemistry calculations of self-assembly 
induced fluorescence mainly deal with absorption spectra. ${ }^{1,19,25}$ Without novel absorption bands, no novel fluorescence bands may appear, and the key to the explanation of the presence of new emission properties is the understanding of long wavelength absorption formation. This paradigm was extended in the recent work of Grisanti et al., where, using ab initio calculations, fluorescence properties of model peptides aggregates were assessed. ${ }^{35}$ Besides the prediction of the emission in the visible spectral range, a conclusion was made on the time-resolved behavior of the aggregation induced fluorescence. Namely, the presence of an ultrafast fluorescence decay and accompanying spectral diffusion, i.e. a gradual fluorescence emission red shift in time, on a sub-picosecond time scale, was described theoretically. This result prompted us to search for experimental indication of an ultrafast component in a self-assembly induced fluorescence system by means of the ultrafast spectroscopy. Taking into account the universal character of this emission, i.e. similarity of its spectral properties for a broad range of systems, we chose to work with a known model system - fibrillar structures that are formed as a result of phenylalanine self-assembly. The fluorescence of phenylalanine was previously characterized, and showed the high fluorescent signal, much higher than the unfortunately too weak signal of cysteine. ${ }^{10}$ Thus, by the example of the fibrils made of phenylalanine, we aim at corroborating the presence of an ultrafast fluorescence decay of the self-assembly induced fluorescence. 

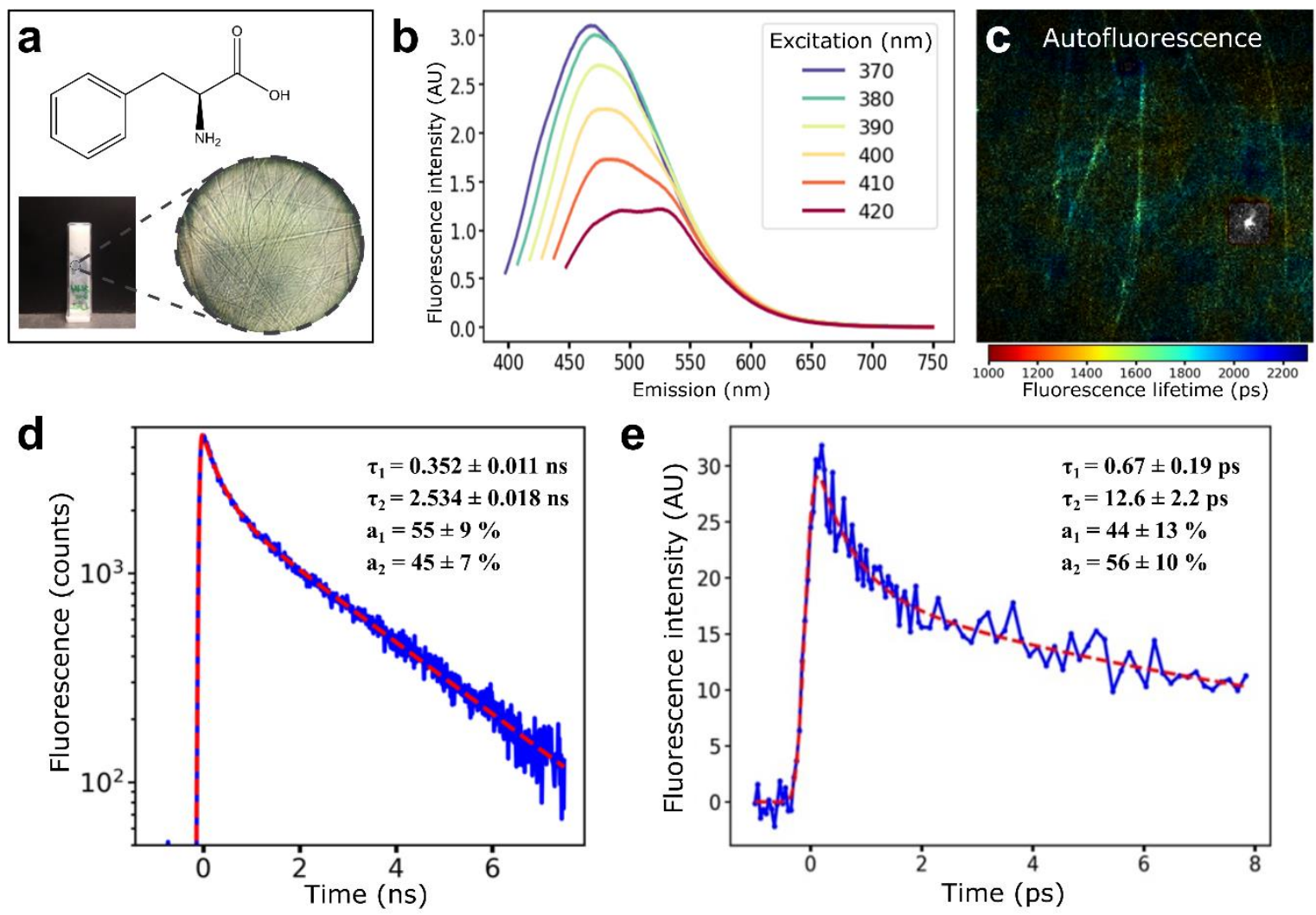

e

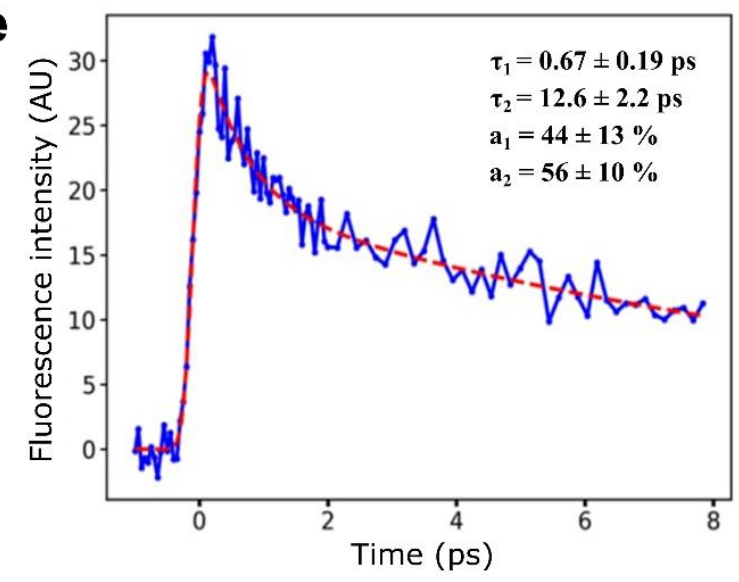

f
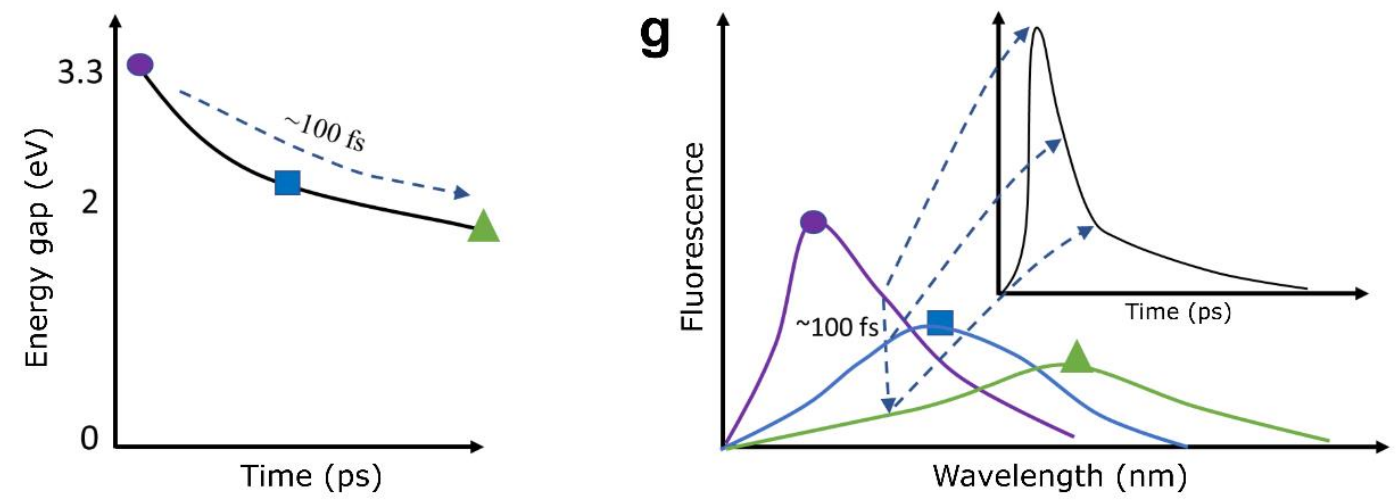

Figure 4. Ultrafast decay. a. Chemical scheme and fibrils of phenylalanine (macroscopic and microscopic photos). b. Fluorescence emission of phenylalanine assemblies at excitations between $370-420 \mathrm{~nm}$. c. Autoluorescence lifetime imaging of phenylalanine fibrils. Excitation was performed in a two-photon regime at $700 \mathrm{~nm}$. d. Fluorescence decay curve of the phenylalanine fibrils measured with (sub)nanosecond resolution. e. Fluorescence decay curve of phenylalanine fibrils measured using fluorescence up-conversion technique. Excitation and emission were set to 380 and $450 \mathrm{~nm}$, respectively. The inset shows the parameters of fluorescence decay obtained using the biexponential decay model $(\mathrm{d}, \mathrm{e})$. f. Schematic decrease in the energy gap between the excited $n \pi^{*}$ and ground state of the model fibrils. g. Schematic of the shift of the aggregation induced fluorescence spectrum with time, demonstrating the presence of the ultrafast decay and spectral migration.

The self-assembly of phenylalanine in aqueous solution was initiated by cooling a supersaturated solution of phenylalanine $(40 \mathrm{mg} / \mathrm{ml})$ at $90{ }^{\circ} \mathrm{C}$ to $20{ }^{\circ} \mathrm{C}$, similar to previous work. ${ }^{10}$ This leads to the formation of elongated fibrillary aggregates, as shown by bright-field microscopy (Figure 4a). The phenylalanine fibrils exhibited relative high fluorescence emission in the visible spectral range, which is absent in the monomeric state of phenylalanine, 
and characterized by the wavelength-dependent Stokes shift, i.e. the spectra measured at longer excitation wavelength exhibited red-shifted emission (Figure $4 b$ ). Such a behaviour of the fluorescence emission band is known as the red-edge excitation shift effect and is characteristic for the self-assembly induced fluorescence of amino acids, peptides and proteins. ${ }^{16,17,27}$ The presence of elongated fibrillar structures was detectable by fluorescence lifetime imaging, without the use of any external dyes, only by using the autofluorescence signal of the sample (Figure 4c). The autofluorescence decay curve obtained for the phenylalanine fibrils, revealed the presence of 0.35 and 2.53 ns decay times after fitting to a biexponential model (Figure 4d). However, the temporal resolution, or the width of the instrument response function (IRF) of the fluorescence lifetime imaging setup, which employs the standard time-correlated single photon counting technique, is $\sim 50 \mathrm{ps}$ and does not allow for detecting any ultrafast decay components, which will be completely masked by the 50 ps IRF. ${ }^{36,37}$ Hence, we have utilized the fluorescence up-conversion technique, which provides for sub-picosecond resolution of fluorescence decay (for more information, see 'Experimental Section').

Using fluorescence up-conversion, it was observed that the phenylalanine fibrils exhibited ultrafast decay lifetime component as fast as $0.67 \mathrm{ps}$. (Figure 4e). The second decay component was characterized by 12.6 ps decay. The presence of the sub-picosecond fluorescence decay is in agreement with the calculation of the recent published work $^{35}$, where the spectral diffusion and relaxation was observed on a few hundred picoseconds time scale.

In the work of Grisanti et al. ${ }^{35}$, the ab initio nonadiabatic dynamics simulations were used to reveal characteristic properties of the excited electronic states of model amyloid-like peptides. It was shown that a visible (blue-green) fluorescence could originate from the $n \pi^{*}$ states localized on the amide groups. The specific structure of amyloids gives rise to stabilization of some of these states, thus lowering the energy gap between the ground and minimum $n \pi^{*}$ state leading to the shift of absorption and fluorescence to the near UV-visible range. Grisanti and co-workers observed that after excitation in the manifold of $n \pi^{*}$ states in 10-15 fs all trajectories 
from different $\mathrm{n} \pi^{*}$ states reached the lowest excited $\mathrm{S}_{1}$ (or $\mathrm{n} \pi^{*}$ min, according to Grisanti et al.) state. In the range of 15-40 fs after excitation UV fluorescence from $\mathrm{S}_{1}$ state is observed and is peaked at $3.3 \mathrm{eV}(\sim 370 \mathrm{~nm})$. After that, on the time scale of $\sim 100-200 \mathrm{fs}$, internal vibration redistribution occurs that leads to additional redshift of the emission spectrum, down to $2.0 \mathrm{eV}$ $(620 \mathrm{~nm})$ (Figure 4f).

This evolution occurs on the time scale of $\sim 100 \mathrm{fs}$ and is accompanied by the gradual shift of the fluorescence emission from the UV to the blue-green region (Figure 4g). By setting the registration emission wavelength to the blue-green region (in our case, to $500 \mathrm{~nm}(\sim 2.5 \mathrm{eV})$ ), the ultrafast relaxation along the potential curve shown in Figure $4 \mathrm{f}$ can be detected. This process is illustrated in the inset of Figure $4 \mathrm{~g}$ and corresponds to the fluorescence decay curve presented in Figure 4e. The ultrafast relaxation occurs on the time scale of $\sim 100 \mathrm{fs}$, corresponding to the non-radiative decay rate of $\sim 10^{12}-10^{13} \mathrm{~s}^{-1}$, which is, however, lower than in the case of monomeric proteins, where the lack of stabilization of the $n \pi *$ states results in the absence of near-UV and visible absorption and blue-green fluorescence. ${ }^{35}$ Moreover, the presence of the ultrafast decay may result in low fluorescence quantum yield of the aggregationinduced emission of proteins $(\sim 0.01) .{ }^{11}$ Further relaxation from the lowest energy $n \pi^{*}{ }_{\min }$ state may then occur on a longer time scale due to the stabilization of the geometry of the aggregate, and this relaxation corresponds to the 1-2 ns fluorescence lifetime, which was observed for the self-assembly induced fluorescence in this paper (Figure 4d) and other previous works. ${ }^{21}$ Hence, by detecting the ultrafast decay of the fibrillar structures formed as the result of phenylalanine self-assembly, the model of Grisanti et al. can be extended from peptides to amino acids, therefore elucidating the origin of their enigmatic fluorescence emission upon packing. ${ }^{35}$

\section{Conclusions}

To conclude, in this work we have studied the optical properties of all 20 coded amino acid powders. Each powder was examined as commercially obtained and also after dissolution, 
reassembly and lyophilization. Surprisingly, lysine exhibited the most intense fluorescence, even though it has no aromatic moieties. Other charged amino acids showed little to no fluorescence. In addition, short-chain derivatives of lysine displayed no correlation between the chain length and their intrinsic fluorescence. This indicates that in some cases, not only the chemical identity of the monomeric molecule dictates the optical properties of the assembly, but also the supramolecular arrangement within the assembly. To substantiate this notion, PXRD analysis together with confocal imaging revealed that the orthorhombic crystal of cysteine is fluorescent, while the monoclinic crystal is not. The recrystallization of the nonfluorescent monoclinic crystals into fluorescent orthorhombic crystals confirms that, in this case, changing the crystal packing is sufficient for conferring optical properties, and that the fluorescence does not stem from contaminations, impurities or oxidation. It is important to note that we do not imply that impurities, oxidation or aromatic interactions are not valid mechanisms that could explain the fluorescence in some cases. However, in addition to those, supramolecular interactions may also affect the optical properties of other biomolecular assemblies, as unambiguously presented here, thus playing a key role in a phenomenon that could be explained by other known mechanisms. In the literature, the phenomenon of selfassembly induced fluorescence is usually addressed for peptides in the context of amyloids optical properties. Several hypotheses have been suggested to explain the mechanism of this effect, and their common motif is that the stabilization of the monomer's structure by hydrogen bonds in a beta-sheet conformation plays a crucial role in the formation of emitting states. Namely, it was proposed that the peptide aggregation induced fluorescence in the blue-green spectral range is due to (i) delocalization of electrons over a network of hydrogen bonds, ${ }^{38}$ (ii) hydrogen bond-mediated interactions between the amide groups, ${ }^{39}$ (iii) proton transfer across hydrogen bonds, ${ }^{1}$ (iv) decrease in the energy gap between the excited and ground states caused by the influence of the hydrogen bonds on the amide group geometry. ${ }^{39}$ The importance and role of the structure for fluorescence formation in aggregates of non-aromatic peptides has been 
recently addressed using ab initio nonadiabatic dynamics simulations of the excited electronic states. $^{39}$ In addition, previous work has shown that low energy optical excitations and subsequent fluorescence can be induced by charge-transfer excitations. ${ }^{20,22,40}$ Specifically, charge transfer excitations involving sulfur atoms of methionine and the positively charged Ntermini of amyloid aggregates were presented. ${ }^{20}$ In the case of cysteine demonstrated in this work, the difference in the molecular packing within the crystal alter the distances between the sulfur atom and the $\mathrm{N}$-termini of the neighboring molecule, which could affect the charge transfer potential. Indeed, these distances are shorter for the orthorhombic crystal in comparison to the monoclinic packing (Figure S2). Overall, on the basis of previous research, it can be summarized that the chromophore responsible for the aggregation induced absorption and fluorescence in the visible range is structure-specific, and can be formed either in the absence or presence of aromatic moieties in peptides. Our results demonstrate that even the simplest and most thoroughly investigated molecular systems, such as amino acids, can still serve as the basis for new, intriguing and unknown phenomena. The reversibility of cysteine fluorescence serves as a strong evidence that the molecular arrangement has a crucial role in the observed optical properties.

The prediction of ultrafast decay by Grisanti et al. was experimentally confirmed, in an aggregation-induced fluorescence model system of phenylalanine fibrils. Specifically, the presence of the ultrafast decay of the blue-green autofluorescence, are in agreement with the hypothesis regarding the governing role of amyloid structure-stabilized $n \pi^{*}$ states in the emission formation. Although the experimental data does not exclude other hypotheses, its relevance to the theoretical calculations can be a step towards understanding the origin of the blue-green emission in amyloids and other systems that recently attracts increased interest, which appear as a result of peptides and amino acid self-assembly. Further understanding of the underlying mechanisms could aid in harnessing the intrinsic properties of supramolecular 
polymers self-assembled by simple and cost-effective building blocks to develop smart optoelectronic materials.

\section{Experimental Section}

\section{Amino Acid Reassembly}

The original samples from Sigma-Aldrich were dissolved in double distilled water, at a relatively high concentration, depending on the water solubility, as detailed in Table S1. The samples were then heated to $90{ }^{\circ} \mathrm{C}$ and vortexed to obtain a clear, transparent solution. The solutions were then incubated at room temperature for a week to allow self-assembly and crystallization. The samples were then lyophilized to remove the water and attain a dry crystalline powder.

\section{Confocal Imaging}

All confocal images were taken using a Leica SP8 Lightning confocal microscope with a Leica Application Suite X (LAS X) software. The samples were excited using a $405 \mathrm{~nm}$ laser, with laser intensity set to $50 \%$ and the gain set to 500 for all images. The emission range was set between $415 \mathrm{~nm}$ to $600 \mathrm{~nm}$. All images were taken at a magnification of $5 \mathrm{X}$.

\section{Image Analysis}

Images were analyzed using Image J. Since we use confocal microscopy, we can assume that the depth $(\mathrm{Z})$ is very small in comparison to the width and height ( $\mathrm{X}$ and $\mathrm{Y})$. Thus, in these images we can consider the areas rather than the volumes. For each image, we divided the number of "fluorescent" pixels by the number of "black" pixels in the corresponding brightfield image. The threshold for "fluorescent" and "black" pixels were identical for all the images in each experimental set.

\section{Powder X-Ray Diffraction}

X-ray diffraction was collected using a Bruker D8 Discover diffractometer with LYNXEYE EX linear position detector. The diffraction pattern to analyze the amino acid structure was performed in a classical $९-\vartheta$ Bragg-Brentano setup. The diffraction patterns were 
corroborated based on the reported phases in the PDF-4-organics-2019 database. As the crystal structure of L-2,4-diaminobutyric acid was not reported in the literature, a full crystal structure determination was performed (CCDC Deposition \# 1990651; Data File S1). The crystalized powder was placed in a $0.7 \mathrm{~mm}$ quartz capillary and a full diffraction pattern was collected between 2 and $50^{\circ}$, step $0.02 \AA$. The capillary setup was employed: Göbels mirrors to obtain a parallel beam, rotating capillary holder. The crystallographic structure determination was performed using the EXPO2014 software. ${ }^{41}$ These EXPO2104 features used were cell indexing (N-TREOR09 algorithm) and the Simulated Annealing Method.

The solution with the lowest cost function was used as model to perform further crystal refinement on the structure using the GSASII software. ${ }^{42}$ The final error indexes were: $\mathrm{wR}=10.9 \%$ and $\mathrm{GoF}=3.77$.

\section{Cysteine Crystallization}

Both cysteine crystal packings were crystallized in double distilled water, at an amino acid concentration of $200 \mathrm{mg} / \mathrm{ml}$. Monoclinic packing was attained as described in the "Amino Acid Reassembly" section above. Orthorhombic packing was attained by incubating the solution over night at $4{ }^{\circ} \mathrm{C}$.

\section{Phenylalanine fibrils sample preparation}

The sample preparation protocol was analogous to the procedures previously used to create self-assembled phenylalanine -aggregates as previously described [Shaham-Niv et al., 2018] using the heat-cool technique. Briefly, L-phenylalanine (Panreac Applichem, CAS 63-91-2, no additional purification) in a concentration of $40 \mathrm{mg} / \mathrm{ml}$ was dissolved in distilled water (Millipore-Q) at $90{ }^{\circ} \mathrm{C}$ (temperature was controlled by the thermostat Qpod 2e, Quantum Northwest, USA) and was stirred for 1 hour using magnetic stirring for complete dissolving. To obtain self-assembled aggregates, the heated phenylalanine solution was cooled to a room temperature of $23-25{ }^{\circ} \mathrm{C}$ under normal conditions in cuvettes or on glass slides, depending on the type of the measurement being performed. 
Steady-state fluorescence measurements of phenylalanine fibrils.

Steady-state fluorescence measurements were performed using the FluoroMax-4 spectrofluorometer (HORIBA Jobin Yvon, Japan). Excitation-emission matrices were measured in the 400-750 $\mathrm{nm}$ emission range with $1 \mathrm{~nm}$ step; the excitation wavelength was varied in the $370-420 \mathrm{~nm}$ range with a $10 \mathrm{~nm}$ step. The spectral widths of the excitation and emission slits were set to $5 \mathrm{~nm}$. The measurements were carried out in a quartz cuvette with an optical path of $1 \mathrm{~cm}$. The measurements were carried out for samples cooled to room temperature.

\section{Fluorescence lifetime microscopy (FLIM)}

Fluorescence lifetime imaging microscopy (FLIM) with multiphoton excitation was performed using a custom-build multiphoton multimodal microscopy setup. Femtosecond optical parametric oscillator TOPOL-1050-C (Avesta, Russia), providing excitation by signal wave in the 680-1000 $\mathrm{nm}$ range, was used as an excitation source. The pulse width of exciting radiation was $\sim 150 \mathrm{fs}$, with frequency of $80 \mathrm{MHz}$, average power at the excitation wavelength on the sample was $1 \mathrm{~mW}$. Scanning over the sample was performed using DSC-120 scan head (Becker\&Hickl, Germany). Imaging was performed using oil immersion Plan Apochromat 60x objective with NA=1.4 (CFI Plan Apochromat Lambda 60×, Nikon, Japan). Fluorescence decay curves were detected using hybrid GaAsP detector HPM-100-40 (Becker\&Hickl, Germany) with sensitivity in $250-720 \mathrm{~nm}$ range and instrument response function characteristic timewidth of 120 ps. To cut off the excitation, a $680 \mathrm{~nm}$ short-pass dielectric filter was used. Both autofluorescence of phenylalanine -fibers and the thioflavin T fluorescence signals were excited at $730 \mathrm{~nm}$.

Fluorescence decay curves were fitted using SPCImage 8.3 software (Becker\&Hickl, Germany) after spatial binning (bin size was equal to 5 for ThT-fluorescence measurements and 10 for autofluorescence measurements) by bi-exponential decay law with respect to instrument response function. Average fluorescence lifetime was calculated as $\tau_{\mathrm{m}}=\left(\mathrm{a}_{1} \tau_{1}+\mathrm{a}_{2}\right.$ 
$\left.\tau_{2}\right) /\left(a_{1}+a_{2}\right)$, where $a_{1}, a_{2}, \tau_{1}, \tau_{2}$ are the amplitudes and lifetimes obtained from fit.

\section{Sub-picosecond fluorescence lifetime measurements.}

Time-resolved fluorescence emission measurements in the sub-picosecond time range were carried out using a commercially available femtosecond fluorescence spectrometry system FOG100 (CDP Systems, Russia). The samples were excited by $100 \mathrm{fs}$ pulses at $380 \mathrm{~nm}$ with a frequency of $80 \mathrm{MHz}$ (second harmonic of Ti:Sapphire oscillator Mai-Tai, Spectra Physics, USA). The fluorescence signal from the sample was focused on a $0.5 \mathrm{~mm} \beta$-barium borate crystal alongside the fundamental beam ( $80 \mathrm{fs}, 760 \mathrm{~nm})$, acting as a gate pulse for the frequency up-conversion. The gate pulse was delayed by an automatically controlled delay stage. The upconverted light was focused onto the entrance slit of the double monochromator (spectral resolution $<1.5 \mathrm{~nm}$ ) and was detected by a photomultiplier tube. The reproducibility of the measurement was checked by 10 times, measuring decay trace. Special rotation cuvette unit was used to avoid photodegradation of the sample.

Similar to steady-state fluorescence and microscopy measurements, a phenylalanine sample in a liquid state, heated to a temperature of $90^{\circ} \mathrm{C}$, was poured into a cuvette and was cooled down to room temperature for 15-20 minutes. The measurements were carried out when the sample was turbid. Fluorescence decay curves were fitted by biexponential decay law with respect to instrument response function fitted as Gaussian function. Data analysis on the subpicosecond decay curves were performed using custom-built Python scripts using LmFit, Matplotlib, Numpy, Pandas libraries.

[CCDC 1990651 contains the supplementary crystallographic data for this paper. These data can be obtained free of charge from The Cambridge Crystallographic Data Centre via www.ccdc.cam.ac.uk/data_request/cif.] 


\section{Supporting Information}

Supporting Information is available online or from the author.

\section{Acknowledgements}

Z.A.A. and T.K. contributed equally to this work. We thanks the all our lab members for the fruitful discussions. Funding: This work was supported by the Israeli National Nanotechnology Initiative and Helmsley Charitable Trust (E.G.), the European Research Council BISON project (E.G.), the Clore Scholarship program and the Marian Gertner Institute (Z.A.A.). The work was supported by the Ministry of Science and Higher Education of the Russian Federation within the framework of state support for the creation and development of World-Class Research Centers "Digital biodesign and personalized healthcare" №075-15-2020-926 (E.S.). We thank members of the Gazit group for the helpful discussions. Author contributions: Z.A.A., T.K., S.S.-N., and E.G. conceived and designed the experiments. Z.A.A., T.K., N.B., R.A., S.S.-N., P.M., M.N.Q., E.P., A.R., I.S., A.H., E.S. and D.L. planned and performed the experiments. Z.A.A., T.K., and E.G wrote the manuscript. D.L. performed PXRD experiments and analysis. All authors discussed the results, provided intellectual input and critical feedback and commented on the manuscript. Competing interests: Authors declare no competing interests.

Received: ((will be filled in by the editorial staff))

Revised: ((will be filled in by the editorial staff)) Published online: ((will be filled in by the editorial staff))

\section{References}

1. Pinotsi, D., Grisanti, L., Mahou, P., Gebauer, R., Kaminski, C.F., Hassanali, A., and Kaminski Schierle, G.S. (2016). Proton transfer and structure-specific fluorescence in hydrogen bond-rich protein structures. J. Am. Chem. Soc. 138, 3046-3057.

2. Bhattacharya, A., Bhowmik, S., Singh, A.K., Kodgire, P., Das, A.K., and Mukherjee, T.K. (2017). Direct Evidence of Intrinsic Blue Fluorescence from Oligomeric Interfaces of Human Serum Albumin. Langmuir 33, 10606-10615. 
3. Pinotsi, D., Buell, A.K., Dobson, C.M., Kaminski Schierle, G.S., and Kaminski, C.F. (2013). A label-free, quantitative assay of amyloid fibril growth based on intrinsic fluorescence. Chembiochem 14, 846-850.

4. Chan, F.T.S., Kaminski Schierle, G.S., Kumita, J.R., Bertoncini, C.W., Dobson, C.M., and Kaminski, C.F. (2013). Protein amyloids develop an intrinsic fluorescence signature during aggregation. Analyst 138, 2156-2162.

5. Ardona, H.A.M., Draper, E.R., Citossi, F., Wallace, M., Serpell, L.C., Adams, D.J., and Tovar, J.D. (2017). Kinetically Controlled Coassembly of Multichromophoric Peptide Hydrogelators and the Impacts on Energy Transport. J. Am. Chem. Soc. 139, 8685-8692.

6. Del Mercato, L.L., Pompa, P.P., Maruccio, G., Della Torre, A., Sabella, S., Tamburro, A.M., Cingolani, R., and Rinaldi, R. (2007). Charge transport and intrinsic fluorescence in amyloid-like fibrils. Proc. Natl. Acad. Sci. U. S. A. 104, 18019-18024.

7. Guerin, S., Stapleton, A., Chovan, D., Mouras, R., Gleeson, M., McKeown, C., Noor, M.R., Silien, C., Rhen, F.M.F.F., Kholkin, A.L.L., et al. (2018). Control of piezoelectricity in amino acids by supramolecular packing. Nat. Mater. 17, 180-186.

8. Ji, W., Xue, B., Arnon, Z.A.Z.A., Yuan, H., Bera, S., Li, Q., Zaguri, D., Reynolds, N.P.N.P., Li, H., Chen, Y., et al. (2019). Rigid Tightly Packed Amino Acid Crystals as Functional Supramolecular Materials. ACS Nano 13, 14477-14485.

9. Makam, P., Yamijala, S.S.R.K.C., Tao, K., Shimon, L.J.W., Eisenberg, D.S., Sawaya, M.R., Wong, B.M., and Gazit, E. (2019). Non-proteinaceous hydrolase comprised of a phenylalanine metallo-supramolecular amyloid-like structure. Nat. Catal. 2, 977-985.

10. Shaham-Niv, S., Arnon, Z.A., Sade, D., Lichtenstein, A., Shirshin, E.A., Kolusheva, S., and Gazit, E. (2018). Intrinsic Fluorescence of Metabolite Amyloids Allows Label-Free Monitoring of Their Formation and Dynamics in Live Cells. Angew. Chemie Int. Ed. 57, 12444-12447. 
11. Niyangoda, C., Miti, T., Breydo, L., Uversky, V., and Muschol, M. (2017). Carbonylbased blue autofluorescence of proteins and amino acids. PLoS One 12, e0176983.

12. Babar, D.G., and Sarkar, S. (2017). Self-assembled nanotubes from single fluorescent amino acid. Appl. Nanosci. 7, 101-107.

13. Banerjee, P., Pyne, A., and Sarkar, N. (2020). Understanding the Self-Assembling Behavior of Biological Building Block Molecules: A Spectroscopic and Microscopic Approach. J. Phys. Chem. B 124, 2065-2080.

14. Zou, L., Harkey, M.R., and Henderson, G.L. (2002). Effects of intrinsic fluorescence and quenching on fluorescence-based screening of natural products. Phytomedicine 9, $263-267$.

15. Lakowicz, J.R., Shen, B., Gryczynski, Z., D’Auria, S., and Gryczynski, I. (2001). Intrinsic fluorescence from DNA can be enhanced by metallic particles. Biochem. Biophys. Res. Commun. 286, 875-879.

16. Berger, O., Adler-Abramovich, L., Levy-Sakin, M., Grunwald, A., Liebes-Peer, Y., Bachar, M., Buzhansky, L., Mossou, E., Forsyth, V.T., Schwartz, T., et al. (2015). Light-emitting self-assembled peptide nucleic acids exhibit both stacking interactions and Watson-Crick base pairing. Nat. Nanotechnol. 10, 353-360.

17. Arnon, Z.A., Berger, O., Aizen, R., Hannes, K., Brown, N., Shimon, L.J.W., and Gazit, E. (2019). Coassembly of Complementary Peptide Nucleic Acid into Crystalline Structures by Microfluidics. Small Methods 3, 1900179.

18. Chen, X., Luo, W., Ma, H., Peng, Q., Yuan, W.Z., and Zhang, Y. (2018). Prevalent intrinsic emission from nonaromatic amino acids and poly(amino acids). Sci. China Chem. 61, 351-359.

19. Stephens, A.D., Qaisrani, M.N., Ruggiero, M.T., Jones, S.T.E., Poli, E., Bond, A.D., Woodhams, P.J., Kleist, E.M., Grisanti, L., Gebauer, R., et al. (2020). Intrinsic fluorescence in non-aromatic peptide structures is induced by collective vibrations, 
charge reorganisation and short hydrogen bonds, as shown in a new glutamine-related structure. bioRxiv, 2020.01.22.915306.

20. Jong, K.H., Azar, Y.T., Grisanti, L., Stephens, A.D., Jones, S.T.E.E., Credgington, D., Kaminski Schierle, G.S., and Hassanali, A. (2019). Low energy optical excitations as an indicator of structural changes initiated at the termini of amyloid proteins. Phys. Chem. Chem. Phys. 21, 23931-23942.

21. Tikhonova, T.N., Rovnyagina, N.R., Zherebker, A.Y., Sluchanko, N.N., Rubekina, A.A., Orekhov, A.S., Nikolaev, E.N., Fadeev, V. V., Uversky, V.N., and Shirshin, E.A. (2018). Dissection of the deep-blue autofluorescence changes accompanying amyloid fibrillation. Arch. Biochem. Biophys. 651, 13-20.

22. Prasad, S., Mandal, I., Singh, S., Paul, A., Mandal, B., Venkatramani, R., and Swaminathan, R. (2017). Near UV-Visible electronic absorption originating from charged amino acids in a monomeric protein. Chem. Sci. 8, 5416-5433.

23. Pansieri, J., Josserand, V., Lee, S.J., Rongier, A., Imbert, D., Sallanon, M.M., Kövari, E., Dane, T.G., Vendrely, C., Chaix-Pluchery, O., et al. (2019). Ultraviolet-visiblenear-infrared optical properties of amyloid fibrils shed light on amyloidogenesis. Nat. Photonics 13, 473-479.

24. Kong, J., Wang, Y., Qi, W., Su, R., and He, Z. (2019). Photo- and Aromatic StackingInduced Green Emissive Peptidyl Nanoparticles for Cell Imaging and Monitoring of Nucleic Acid Delivery. ACS Appl. Mater. Interfaces 11, 15401-15410.

25. Grisanti, L., Pinotsi, D., Gebauer, R., Kaminski Schierle, G.S., and Hassanali, A.A. (2017). A computational study on how structure influences the optical properties in model crystal structures of amyloid fibrils. Phys. Chem. Chem. Phys. 19, 4030-4040.

26. Yamaguchi, S., Kamikubo, H., Kurihara, K., Kuroki, R., Niimura, N., Shimizu, N., Yamazaki, Y., and Kataoka, M. (2009). Low-barrier hydrogen bond in photoactive yellow protein. Proc. Natl. Acad. Sci. U. S. A. 106, 440-444. 
27. Kumar, A., Ahari, D., Priyadarshi, A., Ziauddin Ansari, M., and Swaminathan, R. (2020). Weak Intrinsic Luminescence in Monomeric Proteins Arising from Charge Recombination. J. Phys. Chem. B 124, 2731-2746.

28. Buell, A.K., Dobson, C.M., Knowles, T.P.J., and Welland, M.E. (2010). Interactions between Amyloidophilic Dyes and Their Relevance to Studies of Amyloid Inhibitors. Biophys. J. 99, 3492-3497.

29. Ren, X., Zou, Q., Yuan, C., Chang, R., Xing, R., and Yan, X. (2019). The Dominant Role of Oxygen in Modulating the Chemical Evolution Pathways of Tyrosine in Peptides: Dityrosine or Melanin. Angew. Chemie Int. Ed. 58, 5872-5876.

30. Williams, P.A., Hughes, C.E., Martin, J., Courvoisier, E., Buanz, A.B.M., Gaisford, S., and Harris, K.D.M. (2016). Understanding the Solid-State Hydration Behavior of a Common Amino Acid: Identification, Structural Characterization, and Hydration/Dehydration Processes of New Hydrate Phases of 1-Lysine. J. Phys. Chem. C 120, 9385-9392.

31. Chiba, A., Ueki, T., Ashida, T., Sasada, Y., and Kakudo, M. (1967). The crystal structure of L-ornithine hydrochloride. Acta Crystallogr. 22, 863-870.

32. Marčeková, M., Gerža, P., Šoral, M., Moncol, J., Berkeš, D., Kolarovič, A., and Jakubec, P. (2019). Crystallization Does It All: An Alternative Strategy for Stereoselective Aza-Henry Reaction. Org. Lett. 21, 4580-4584.

33. Kerr, K.A., and Ashmore, J.P. (1973). Structure and conformation of orthorhombic Lcysteine. Acta Crystallogr. Sect. B Struct. Crystallogr. Cryst. Chem. 29, 2124-2127.

34. Harding, M.M., and Long, H.A. (1968). The crystal and molecular structure of Lcysteine. Acta Crystallogr. Sect. B Struct. Crystallogr. Cryst. Chem. 24, 1096-1102.

35. Grisanti, L., Sapunar, M., Hassanali, A., and Došlić, N. (2020). Toward Understanding Optical Properties of Amyloids: A Reaction Path and Nonadiabatic Dynamics Study. J. Am. Chem. Soc. 142, 18042-18049. 
36. Rovnyagina, N.R., Budylin, G.S., Vainer, Y.G., Tikhonova, T.N., Vasin, S.L., Yakovlev, A.A., Kompanets, V.O., Chekalin, S. V., Priezzhev, A. V., and Shirshin, E.A. (2020). Fluorescence lifetime and intensity of thioflavin T as reporters of different fibrillation stages: Insights obtained from fluorescence up-conversion and particle size distribution measurements. Int. J. Mol. Sci. 21, 1-16.

37. Rovnyagina, N.R., Tikhonova, T.N., Kompanets, V.O., Sluchanko, N.N., Tugaeva, K. V., Chekalin, S. V., Fadeev, V. V., Lademann, J., Darvin, M.E., and Shirshin, E.A. (2019). Free and bound Thioflavin T molecules with ultrafast relaxation: Implications for assessment of protein binding and aggregation. Laser Phys. Lett. 16, 075601.

38. Shukla, A., Mukherjee, S., Sharma, S., Agrawal, V., Radha Kishan, K.., Guptasarma, P., Kishan, K.V.R., and Guptasarma, P. (2004). A novel UV laser-induced visible blue radiation from protein crystals and aggregates: scattering artifacts or fluorescence transitions of peptide electrons delocalized through hydrogen bonding? Arch. Biochem. Biophys. 428, 144-153.

39. Ye, R., Liu, Y., Zhang, H., Su, H., Zhang, Y., Xu, L., Hu, R., Kwok, R.T.K., Wong, K.S., Lam, J.W.Y., et al. (2017). Non-conventional fluorescent biogenic and synthetic polymers without aromatic rings. Polym. Chem. 8, 1722-1727.

40. Mandal, I., Manna, S., and Venkatramani, R. (2019). UV-Visible Lysine-Glutamate Dimer Excitations in Protein Charge Transfer Spectra: TDDFT Descriptions Using an Optimally Tuned CAM-B3LYP Functional. J. Phys. Chem. B 123, 10967-10979.

41. Altomare, A., Cuocci, C., Giacovazzo, C., Moliterni, A., Rizzi, R., Corriero, N., and Falcicchio, A. (2013). EXPO2013: A kit of tools for phasing crystal structures from powder data. J. Appl. Crystallogr. 46, 1231-1235.

42. Toby, B.H., and Von Dreele, R.B. (2013). GSAS-II: The genesis of a modern opensource all purpose crystallography software package. J. Appl. Crystallogr. 46, 544-549.

43. Wilson, C.C., Myles, D., Ghosh, M., Johnson, L.N., and Wang, W. (2005). Neutron 
diffraction investigations of 1- and d-alanine at different temperatures: the search for structural evidence for parity violation. New J. Chem. 29, 1318.

44. Mazumdar, S.K., Venkatesan, K., Mez, H.-C., and Donohue, J. (1969). The crystal structure of 1-arginine hydrochloride. Zeitschrift für Krist. 130, 328-339.

45. Verbist, J.J., Lehmann, M.S., Koetzle, T.F., and Hamilton, W.C. (1972). Precision neutron diffraction structure determination of protein and nucleic acid components. VI. The crystal and molecular structure of the amino acid L-asparagine monohydrate. Acta Crystallogr. Sect. B Struct. Crystallogr. Cryst. Chem. 28, 3006-3013.

46. Yamada, K., Hashizume, D., Shimizu, T., and Yokoyama, S. (2007). L-Asparagine. Acta Crystallogr. Sect. E Struct. Reports Online 63, 3802-3803.

47. Derissen, J.L., Endeman, H.J., and Peerdeman, A.F. (1968). The crystal and molecular structure of L-aspartic acid. Acta Crystallogr. Sect. B Struct. Crystallogr. Cryst. Chem. 24, 1349-1354.

48. Koetzle, T.F., Frey, M.N., Lehmann, M.S., and Hamilton, W.C. (1973). Precision neutron diffraction structure determination of protein and nucleic acid components. XIII. Molecular and crystal structure of the amino acid L-glutamine. Acta Crystallogr. Sect. B Struct. Crystallogr. Cryst. Chem. 29, 2571-2575.

49. Lehmann, M.S., Koetzle, T.F., and Hamilton, W.C. (1972). Precision neutron diffraction structure determination of protein and nucleic acid components. VIII: the crystal and molecular structure of the $\beta$-form of the amino acidl-glutamic acid. J. Cryst. Mol. Struct. 2, 225-233.

50. Almlöf, J., Kvick, Å., and Thomas, J.O. (1973). Hydrogen bond studies. 77. Electron density distribution in $\alpha$-glycine: $\mathrm{X}-\mathrm{N}$ difference Fourier synthesis vs ab initio calculations. J. Chem. Phys. 59, 3901-3906.

51. Kvick, Å.;., Canning, W.M., Koetzle, T.F., and Williams, G.J.B. (1980). An experimental study of the influence of temperature on a hydrogen-bonded system: the 
crystal structure of $\gamma$-glycine at $83 \mathrm{~K}$ and $298 \mathrm{~K}$ by neutron diffraction. Acta Crystallogr. Sect. B Struct. Crystallogr. Cryst. Chem. 36, 115-120.

52. Oda, K., and Koyama, H. (1972). A refinement of the crystal structure of histidine hydrochloride monohydrate. Acta Crystallogr. Sect. B 28, 639-642.

53. Torii, K., and Iitaka, Y. (1971). The crystal structure of L-isoleucine. Acta Crystallogr. Sect. B Struct. Crystallogr. Cryst. Chem. 27, 1317-1326.

54. Coll, M., Solans, X., Font-Altaba, M., and Subirana, J.A. (1986). Structure of Lleucine: a redetermination. Acta Crystallogr. Sect. C Cryst. Struct. Commun. 42, 599601.

55. Torii, K., and Iitaka, Y. (1973). Crystal structures and molecular conformations of Lmethionine and L-norleucine. Acta Crystallogr. Sect. B Struct. Crystallogr. Cryst. Chem. 29, 2799-2807.

56. King, M.D., Blanton, T.N., and Korter, T.M. (2012). Revealing the true crystal structure of L-phenylalanine using solid-state density functional theory. Phys. Chem. Chem. Phys. 14, 1113-1116.

57. Janczak, J., and Luger, P. (1997). L-Proline Monohydrate at 100 K. Acta Crystallogr. Sect. C Cryst. Struct. Commun. 53, 1954-1956.

58. Kayushina RL, V.B.R. (1965). Opredepenie strukturi L-prolina. Kristallografiya 10, $833-844$.

59. Kistenmacher, T.J., Rand, G.A., and Marsh, R.E. (1974). Refinements of the crystal structures of DL-serine and anhydrous L-serine. Acta Crystallogr. Sect. B Struct. Crystallogr. Cryst. Chem. 30, 2573-2578.

60. Frey, M.N., Lehmann, M.S., Koetzle, T.F., and Hamilton, W.C. (1973). Precision neutron diffraction structure determination of protein and nucleic acid components. XI. Molecular configuration and hydrogen bonding of serine in the crystalline amino acids L-serine monohydrate and DL-serine. Acta Crystallogr. Sect. B Struct. Crystallogr. 
Cryst. Chem. 29, 876-884.

61. Ramanadham, M., Sikka, S.K., and Chidambaram, R. (1973). Structure determination of Ls-threonine by neutron diffraction. Pramana 1, 247-259.

62. Khawas, B., and Krishna Murti, G.S.R. (1969). On the unit-cell dimensions and space group of L-tyrosine and L-tryptophane. Acta Crystallogr. Sect. B Struct. Crystallogr. Cryst. Chem. 25, 1006-1009.

63. Mostad, A., Rømming, C., Graver, H., Husebye, S., Klæboe, P., and Swahn, C.-G. (1973). Crystal Structure of DL-Tyrosine. Acta Chem. Scand. 27, 401-410.

64. Torii, K., and Iitaka, Y. (1970). The crystal structure of L-valine. Acta Crystallogr. B. $26,1317-1326$. 
The basis for aggregation-induced fluorescence in the visible range is elusive. Here, we systematically examine the optical properties of all coded amino acids. Several amino acids show intense visible fluorescence. Cysteine exhibit fluorescence that is dependent on the crystal packing, as repeated rearrangements between crystallographic polymorphs resulted in a reversible on-off optical transition. Our study corroborates that electronic interactions between non-absorbing molecules at the monomeric state may result in reversible optically-active states by the formation of supramolecular fluorophores.

\section{On-off transition and ultrafast decay of amino acid luminescence driven by modulation} of supramolecular packing

Zohar A. Arnon, Topaz Kreiser, Boris Yakimov, Noam Brown, Ruth Aizen, Shira ShahamNiv, Pandeeswar Makam, Muhammad Nawaz Qaisrani, Emiliano Poli, Antonella Ruggiero, Inna Slutsky, Ali Hassanali, Evgeny Shirshin, Davide Levy and Ehud Gazit*

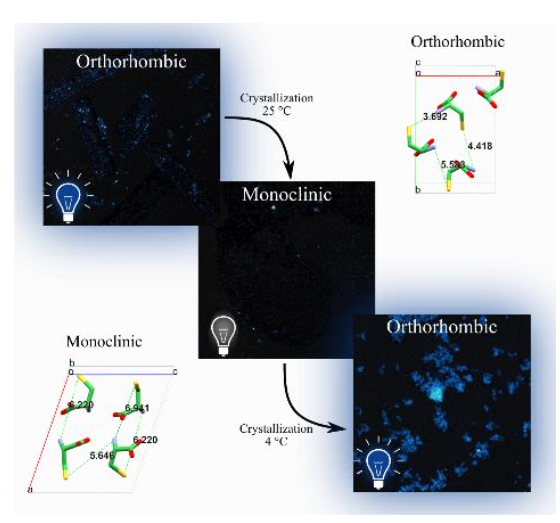




\section{Supporting Information}

\section{On-off transition and ultrafast decay of amino acid luminescence driven by modulation of supramolecular packing}

Zohar A. Arnon, Topaz Kreiser, Boris Yakimov, Noam Brown, Ruth Aizen, Shira Shaham-Niv, Pandeeswar Makam, Muhammad Nawaz Qaisrani, Emiliano Poli, Antonella Ruggiero, Inna Slutsky, Ali Hassanali, Evgeny Shirshin, Davide Levy and Ehud Gazit*

\section{Table S1.}

\begin{tabular}{|l|l|l|l|}
\hline Amino acid & Product name & Product Number & $\begin{array}{l}\text { DR concentration } \\
{[\mathbf{m g} / \mathbf{m l}]}\end{array}$ \\
\hline Alanine & L-Alanine & A7627 & 200 \\
\hline Arginine & L-Arginine monohydrochloride & 11039 & 1000 \\
\hline Aspargine & L-Aspargine & A-0884 & 200 \\
\hline Aspartic acid & L-Aspartic acid & A9256 & 10 \\
\hline Cysteine & L-Cysteine & 168179 & 200 \\
\hline Glutamine & L-Glutamine & G-3126 & 72 \\
\hline Glutamic acid & L-Glutamic acid & G1251 & 17.2 \\
\hline Glycine & L-Glycine & G7126 & 400 \\
\hline Histidine & L-Histidine monohydrochloride monohydrate & H8125 & 200 \\
\hline Isoleucine & L-Isoleucine & I2752 & 82.4 \\
\hline Leucine & L-Leucine & L8000 & 30 \\
\hline Lysine & L-Lysine & L5501 & 200 \\
\hline Methionine & L-Methionine & M9625 & 100 \\
\hline Phenylalanine & L-Phenylalanine & P2126 & 59.2 \\
\hline Proline & L-Proline & P-0380 & 200 \\
\hline Serine & L-Serine & S4311 & 500 \\
\hline Threonine & L-Threonine & T8625 & 200 \\
\hline Tryptophan & L-Tryptophan & T0254 & 26.8 \\
\hline Tyrosine & L-Tyrosine & T3754 & 2 \\
\hline Valine & L-Valine & V0500 & 100 \\
\hline
\end{tabular}

Table S1. List of amino acids, as purchased from Sigma-Aldrich. DR concentration denotes the concentration used to dissolve and reassemble the amino acid. 
Table S2.

\begin{tabular}{|c|c|c|}
\hline Amino Acid & Sigma Powder (OS) & Lyophilized Powder (DR) \\
\hline Alanine & $\begin{array}{l}\text { L-Alanine Orthorhombic } \\
\text { PDF 02-089-6379 }\end{array}$ & $\begin{array}{l}\text { L-Alanine Orthorhombic } \\
\text { PDF 02-089-6379 }\end{array}$ \\
\hline Arginine & $\begin{array}{l}\text { L-Arginine Hydrochloride Monoclinic } \\
\text { PDF 02-063-2292 }\end{array}$ & $\begin{array}{l}\text { L-Arginine Hydrochloride Monoclinic } \\
\text { PDF 02-063-2292 }\end{array}$ \\
\hline Asparagine & $\begin{array}{c}\text { L-Asparagine Monohydrate } \\
\text { PDF 02-060-1510; } \\
\text { L-Aspargine } \\
\text { PDF 02-095-9836 } \\
\end{array}$ & $\begin{array}{c}\text { L-Asparagine Monohydrate } \\
\text { PDF 02-060-1510 }\end{array}$ \\
\hline Aspartic acid & $\begin{array}{c}\text { L-Aspartic Acid } \\
\text { PDF 02-063-2298 } \\
\end{array}$ & $\begin{array}{c}\text { L-Aspartic Acid } \\
\text { PDF 02-063-2298 }\end{array}$ \\
\hline Cysteine & $\begin{array}{l}\text { L-Cysteine Orthorhombic } \\
\text { PDF 02-073-1763 }\end{array}$ & $\begin{array}{l}\text { L-Cysteine Monoclinic } \\
\text { PDF 02-063-2319 }\end{array}$ \\
\hline Glutamine & $\begin{array}{c}\text { L-Glutamine } \\
\text { PDF 02-063-016048 }\end{array}$ & $\begin{array}{c}\text { L-Glutamine } \\
\text { PDF 02-063-0160 }\end{array}$ \\
\hline Glutamic acid & $\begin{array}{c}\text { L-Glutamic acid } \\
\text { PDF 02-063-2343 }\end{array}$ & $\begin{array}{c}\text { L-Glutamic acid } \\
\text { PDF 02-063-2343 }\end{array}$ \\
\hline Glycine & $\begin{array}{l}\text { L-Glycine Monoclinic } \\
\text { PDF 02-063-0180; } \\
\text { L-Glycine Hexagonal } \\
\text { PDF 02-063-018151 } \\
\end{array}$ & $\begin{array}{c}\text { L-Glycine Monoclinic } \\
\text { PDF 02-063-0180; } \\
\text { L-Glycine Hexagonal } \\
\text { PDF 02-063-0181 } \\
\end{array}$ \\
\hline Histidine & $\begin{array}{l}\text { L-Histidine Hydrochloride Monoclinic } \\
\text { PDF 02-063-0694 } \\
\end{array}$ & $\begin{array}{l}\text { L-Histidine Hydrochloride Monoclinic } \\
\text { PDF 02-063-0694 }\end{array}$ \\
\hline Isoleucine & $\begin{array}{c}\text { L-Isoleucine Monoclinic } \\
\text { PDF 02-063-2412 } \\
\end{array}$ & $\begin{array}{c}\text { L-Isoleucine Monoclinic } \\
\text { PDF 02-603-2412 } \\
\end{array}$ \\
\hline Leucine & $\begin{array}{l}\text { L-Leucine Monoclinic } \\
\text { PDF 02-063-2332 }\end{array}$ & $\begin{array}{l}\text { L-Leucine Monoclinic } \\
\text { PDF 02-063-2332 }\end{array}$ \\
\hline Lysine & $\begin{array}{c}\text { L-Lysine Hemihydrate } \\
\text { CCDC } 1488097^{30}\end{array}$ & $\begin{array}{c}\text { L-Lysine Hemihydrate } \\
\text { CCDC } 1488097\end{array}$ \\
\hline Methionine & $\begin{array}{l}\text { L-Methionine Monoclinic } \\
\text { PDF 02-063-2419 }\end{array}$ & $\begin{array}{l}\text { L-Methionine Monoclinic } \\
\text { PDF 02-063-2419 } \\
\end{array}$ \\
\hline Phenylalanine & $\begin{array}{c}\text { L-Phenylalanine } \\
\text { PDF 00-063-1180 }\end{array}$ & $\begin{array}{l}\text { L-Phenylalanine } \\
\text { PDF 00-063-1180 }\end{array}$ \\
\hline Proline & $\begin{array}{l}\text { L-Proline Monohydrate Monoclinic } \\
\text { PDF 02-082-9045; }{ }^{57} \\
\text { L-Proline Orthorhombic } \\
\text { PDF 02-063-7646 } \\
\end{array}$ & $\begin{array}{l}\text { L-Proline Monohydrate Monoclinic } \\
\text { PDF 02-082-9045 }\end{array}$ \\
\hline Serine & $\begin{array}{c}\text { L-Serine Orthorhombic } \\
\text { PDF 02-063-2442 } \\
\end{array}$ & $\begin{array}{l}\text { L-Serine Monohydrate Orthorhombic } \\
\text { PDF 02-063-2444 }\end{array}$ \\
\hline Threonine & $\begin{array}{l}\text { L-Threonine Orthorhombic } \\
\text { PDF 02-063-2447 }\end{array}$ & $\begin{array}{c}\text { L-Threonine Orthorhombic } \\
\text { PDF 02-063-2447 }\end{array}$ \\
\hline Tryptophan & $\begin{array}{c}\text { L-Tryptophan } \\
\text { PDF 00-025-1960 }\end{array}$ & $\begin{array}{c}\text { L-Tryptophan } \\
\text { PDF 00-025-1960 }\end{array}$ \\
\hline Tyrosine & $\begin{array}{l}\text { L-Tyrosine Orthorhombic } \\
\text { PDF 02-063-2456 }\end{array}$ & $\begin{array}{l}\text { L-Tyrosine Orthorhombic } \\
\text { PDF 02-063-2456 }\end{array}$ \\
\hline Valine & $\begin{array}{c}\text { L-Valine } \\
\text { PDF 02-063-2485 } \\
\end{array}$ & $\begin{array}{c}\text { L-Valine } \\
\text { PDF 02-063-2485 }\end{array}$ \\
\hline L-ornithine & L-Ornithine hydrochloride ${ }^{31}$ & - \\
\hline $\begin{array}{c}\text { L-2,4- } \\
\text { diaminobutyric } \\
\text { acid }\end{array}$ & $\begin{array}{c}\text { L-2,4-Diaminobutyric Acid Hydrocholride } \\
\text { CCDC Deposition \# } 1990651\end{array}$ & - \\
\hline $\begin{array}{c}\text { DL-2,3- } \\
\text { diaminopropionic } \\
\text { acid } \\
\end{array}$ & (R)-2,3-diammoniopropanoate chloride ch $^{32}$ & - \\
\hline
\end{tabular}

Table S2. List of PXRD references (Powder Diffraction File, PDF) for the original sigma powders and the dissolved, 
reassembled and lyophilized samples. L-Lysine Hemidydrate does not have a PDF number, hence, we include the Cambridge Crystallographic Data Centre (CCDC) number.

Table S3.

\begin{tabular}{|c|c|}
\hline Orthorhombic & Monoclinic \\
\hline $2.763(\mathrm{x} 8), 2.79(\mathrm{x} 7), 3.013(\mathrm{x} 7)$ & $2.721(\mathrm{x} 3), 2.765(\mathrm{x} 2), 2.886(\mathrm{x} 4)$, \\
& $2.914(\mathrm{x} 8), 2.97(\mathrm{x} 4)$ \\
\hline
\end{tabular}

Table S3. List of Hydrogen bonds lengths $(\AA)$ for both the orthorhombic and monoclinic crystal packings.

\section{Figure S1}
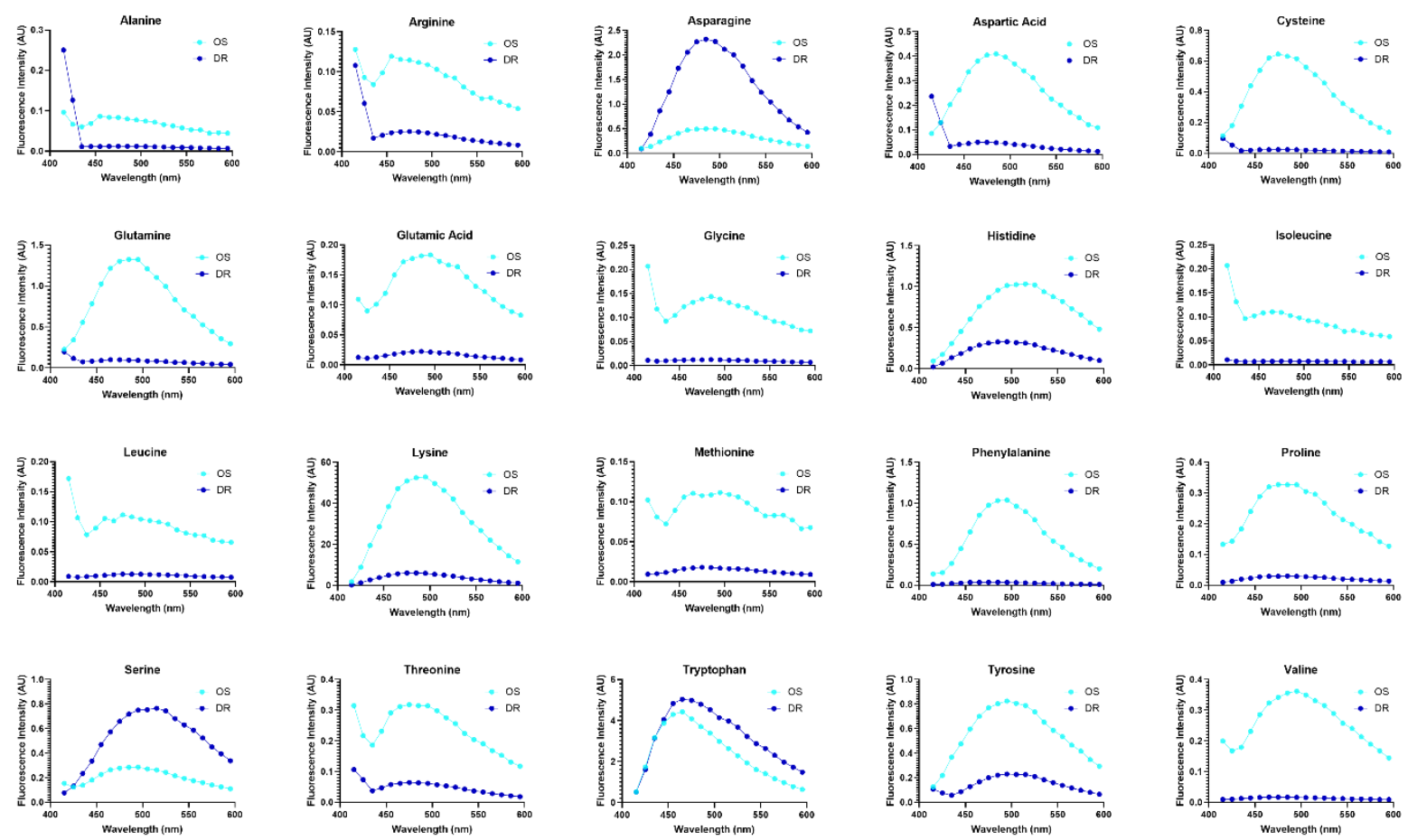

Figure S1. Fluorescence spectra of all 20 amino acids. The fluorescence intensity of all samples as a function of the emission wavelength (excitation at $405 \mathrm{~nm}$ ), obtained by confocal microscopy. 


\section{Figure S2}

\section{Orthorhombic}

\section{Monoclinic}
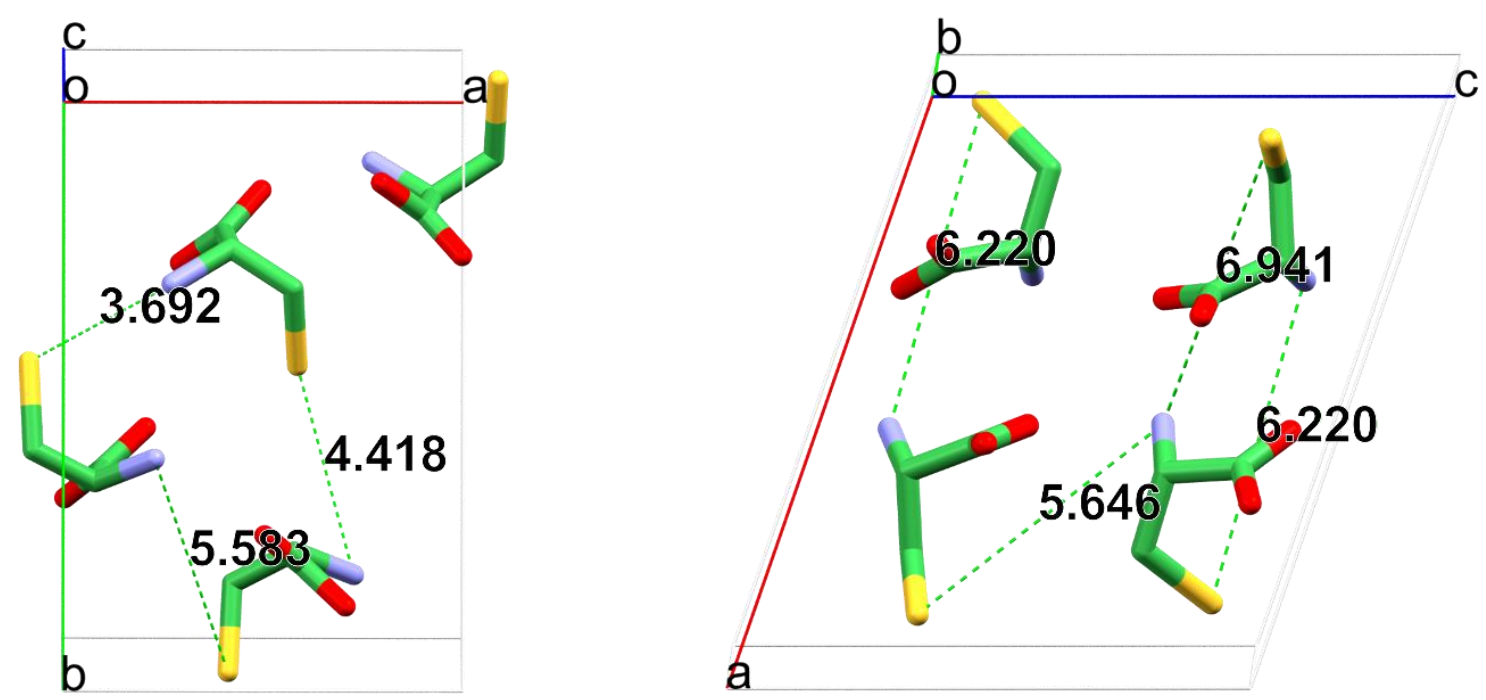

Figure S2. Cysteine crystal structure distances. The measured distances between sulfide atoms and their closest neighboring molecule's amide. Distances displayed are in $\AA$ units. 
bioRxiv preprint doi: https://doi.org/10.1101/2021.03.23.436384; this version posted March 23, 2021. The copyright holder for this preprint (which was not certified by peer review) is the author/funder. All rights reserved. No reuse allowed without permission.

\section{Data S1.}

Crystallographic Information File (CIF) for the crystal structure of L-2,4-diaminobutyric acid (CCDC Deposition \# 1990651). 